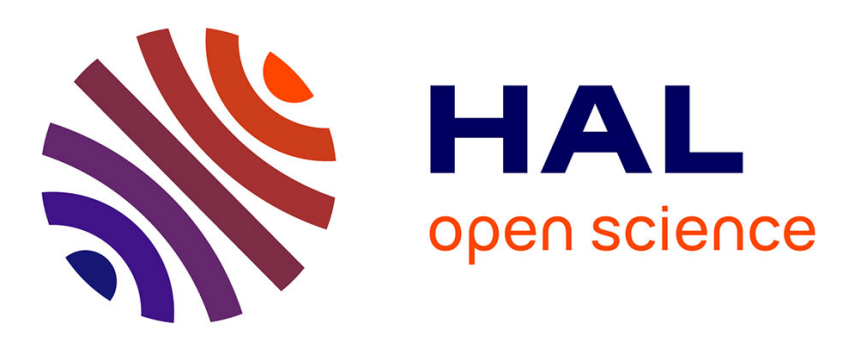

\title{
Multi-Games for LTE and WiFi Coexistence over Unlicensed Channels
}

\author{
Kenza Hamidouche, Saad Walid, Merouane Debbah
}

\section{To cite this version:}

Kenza Hamidouche, Saad Walid, Merouane Debbah. Multi-Games for LTE and WiFi Coexistence over Unlicensed Channels. Network Games, Control, and Optimization Proceedings of NETGCOOP 2016, Avignon, France, 2017. hal-01784888

\section{HAL Id: hal-01784888 \\ https://hal-centralesupelec.archives-ouvertes.fr/hal-01784888}

Submitted on 12 Jul 2018

HAL is a multi-disciplinary open access archive for the deposit and dissemination of scientific research documents, whether they are published or not. The documents may come from teaching and research institutions in France or abroad, or from public or private research centers.
L'archive ouverte pluridisciplinaire HAL, est destinée au dépôt et à la diffusion de documents scientifiques de niveau recherche, publiés ou non, émanant des établissements d'enseignement et de recherche français ou étrangers, des laboratoires publics ou privés. 


\title{
Multi-Games for LTE and WiFi Coexistence over Unlicensed Channels
}

\author{
Kenza Hamidouche ${ }^{1,2}$, Walid Saad ${ }^{2}$, and Mérouane Debbah ${ }^{1,3}$
}

\begin{abstract}
I
In this paper, a novel framework for optimizing the coexistence between LTE and WiFi over unlicensed bands, is proposed. The problem is modeled using the framework of multi-game theory in which the WiFi users (WUs) are considered as leaders and the small base stations (SBSs) as followers. This multi-game framework encompasses two games of different types. In this regard, the competition between the WUs to access the unlicensed channels is formulated as a one-sided matching game while the power allocation problem of the SBSs is formulated as a noncooperative game. In this multi-game, the SBSs anticipate the channel allocation on the WiFi network and adapt their strategies accordingly while the WUs predict the power allocation of the SBSs. For the latter, the existence of a unique Debreu equilibrium is proved while for the matching game the existence of core stable outcome is shown and a decentralized algorithm that converges to the stable outcome is proposed.
\end{abstract}

\section{Introduction}

One promising approach to overcome the scarcity of the radio spectrum is to enable tomorrow's small cell networks (SCNs) to exploit simultaneously their licensed cellular bands along with the unlicensed, WiFi bands[1, 2, 3, 4, 5].

Although offloading part of the LTE traffic to the unlicensed bands can considerably increase the performance of cellular networks, the disparity of the medium access protocols that are used by the WiFi users (WUs) and dual mode SBSs, rise new challenges. In fact, the access to the spectrum in $\mathrm{WiFi}$ is based on carrier sense multiple access with collision avoidance (CSMA/CA) while LTE uses orthogonal frequency division multiple access (OFDMA). In many countries, there are no imposed regulations and the operators need to develop new scheduling mechanisms known as LTE-U to extend the LTE into the unlicensed channels.

In this regard, many works have proposed and analyzed new mechanisms for the deployment of LTE-U over unlicensed channels. The work in [6] formulated the unlicensed spectrum allocation problem with uplink-downlink decoupling as a noncooperative game in which the SBSs are the players that select the unlicensed channels over which they serve their users. The authors in [7] formulated the unlicensed

\footnotetext{
${ }^{1}$ LANEAS Group, CentraleSupélec, Université Paris-Saclay, 3 rue Joliot-Curie, 91192 Gif-surYvette, France, e-mail: kenza.hamidouche@centralesupelec.fr

2 Wireless@VT, Bradley Department of Electrical and Computer Engineering, Virginia Tech, USA, e-mail: walids@vt.edu

3 Mathematical and Algorithmic Sciences Lab, Huawei France R\&D, France, e-mail: merouane.debbah@huawei.com
} 
spectrum allocation problem as a student-project matching problem with externalities.The work in [8] proposed a distributed traffic offloading scheme for LTE-U scenarios with a single base station. In [9], the authors proposed hyper-access points (HAP) in which the functions of both an LTE SBS and a Wi-Fi access point (AP) are combined in the same HAP. In [10], the authors analyze the performance dynamics of integrated LTE-U and WiFi when sharing the unlicensed bands.

Despite being interesting, in all of these existing works, the WUs are either assumed to transmit on fixed channels or the allocation of the unlicensed channels to the WUs is determined without accounting for the existing SBSs.

The main contribution of this paper is to propose a new unlicensed spectrum allocation approach that accounts for the impact of the SBSs' transmit power and the assigned channels to each of the WUs, on one another. We formulate a multigame [5] with two different types of games in which the WUs are considered as leaders and the dual-mode SBSs are the followers. On the followers side, the power allocation problem at the SBSs on the unlicensed channels is formulated as a noncooperative. On the other hand, we formulate the channels allocation problem as a one-sided matching game with externalities [11], in which the WUs predict the transmit power of the SBSs and autonomously select the channel over which they serve their traffic. For the formulated multi-game, we prove that there exists a unique Debreu equilibrium (DE) for the power allocation problem. Moreover, we propose a new distributed matching algorithm for the assignment of the WUs to the unlicensed channels and prove that the proposed algorithm converges to a unique stable matching that is in the core.

\section{System Model}

Consider a wireless network composed of a set $\mathcal{N}$ of $N$ users served by a set $\mathcal{S}$ of $S$ dual-mode small base stations (SBSs) and a set $\mathcal{W}$ of $W$ WiFi users (WUs). The SBSs and WUs can transmit their content over a set $\mathcal{C}$ of $C$ unlicensed channels. Here, each of the channels can be allocated to multiple WUs at a given time but only one WU can transmit based on the LBT scheduling scheme. These same bands when available, are allocated to the SBSs allowing them to aggregate LTE and LTEUnlicensed (LTE-U) to improve the quality-of-service (QoS) of the served users.

The SBSs only serve the downlink traffic over unlicensed channels while in the WiFi network, both downlink and uplink transmissions can occur. The set of $W$ WUs is denoted $\mathcal{W}$. The achievable throughput by a WiFi user $w$ in the network depends on the number of active WUs and SBSs that transmit over the same unlicensed channel $c \in \mathcal{C}$, which we denote $R_{w}\left(W_{c}, S_{c}\right)$, where $W_{c}$ is the number of active WUs on channel $c$ and $S_{c}$ is the number of SBSs that transmit over channel $c$ with a positive transmit power. The faction of time each WU $w$ uses a channel $c$, also known as the channel busy time, is given by [12]:

$$
t_{w c}\left(W_{c}, S_{c}\right)=\frac{l_{w c}}{R_{w}\left(W_{c}, S_{c}\right)}+\gamma_{w c},
$$


where $l_{w}$ is the size of transmitted file by the WU $w$ and $\gamma_{w c}$ is the channel access overhead and the protocol overhead which depends on the type of the file. The WiFi network throughput $R\left(W_{c}, S_{c}\right)$ is given by:

$$
R_{w}\left(W_{c}, S_{c}\right)=\frac{P_{w}^{s} \bar{L}}{P_{w}^{s} T^{s}+P^{c} T^{c}+P^{\sigma} T^{\sigma}},
$$

where $P_{w}^{s}=\tau_{w} \prod_{i \neq w}^{W_{c}+S_{c}}\left(1-\tau_{i}\right)$ is the probability of a successful transmission which corresponds to the probability of having only one transmission over the channel and $\tau_{w}$ is the probability of transmission by a given WU w. $\bar{L}$ is the average size of a packet file, $T^{s}$ is the average time needed to transmit a packet of size $\bar{L}, P^{\sigma}=$ $\prod_{i=1}^{W_{c}}\left(1-\tau_{i}\right)$ is the probability of the channel being idle, $T^{\sigma}$ is the duration of the idle period, $P^{c}$ is the probability of collision, and $T^{c}$ is the average time spent in the collision. The WUs detct collisions based on the power or interference they sense on the unlicensed channels. In fact, if the sensed interference from all other WUs and SBSs over a channel $c$ exceeds a given threshold $I_{t h}$, the WUs consider the channel as busy and back-off. For the SBSs, the achievable physical throughput by a user $j$ that is served by SBS $i$ is given by:

$$
\begin{aligned}
& R_{i c}=\omega_{c} \log \left(1+\frac{p_{i c}\left|h_{i c}\right|^{2}}{\sigma^{2}+\sum_{l \in \mathcal{S} \backslash i} p_{l c}\left|h_{l c}\right|^{2}+\sum_{j \in \mathcal{W}} \alpha_{j c} p_{j c}\left|h_{j c}\right|^{2}}\right),
\end{aligned}
$$

where $p_{i c}$ is the transmission power of SBS/WU $i$ through channel $c,\left|h_{i c}\right|$ is the channel gain of SBS/WU $i$ over channel $c$, and $\omega_{c}$ is the bandwidth of band $c . \alpha_{j c}$ is a boolean that is related to the CSMA/CA transmission mode indicating that WU $j$ is transmitting on channel $c$ when $\alpha_{j c}=1$, and $\alpha_{j c}=0$ otherwise.

\section{Game Formulation and Analysis}

To ensure a harmonious coexistence between the SBSs and the WUs, we formulate a multi-game which is a new game-theoretic framework that we introduced in [5]. A multi-game is a hierarchical game in which multiple interconnected sub-games are formulated. Different from classical Stackelberg games, the games can be of different types and the sets of players are not necessarily disjoint. In our context, the WUs are regarded as leaders in the formulated multi-game, that have the priority in using the unlicensed spectrum. On the other hand, the SBSs are considered as followers that adapt their traffic on unlicensed spectrum based on the leaders' traffic load.

\subsection{Followers' Game}

A set of SBSs compete over the unlicensed channels to transmit their content over the unlicensed bands with a predefined QoS requirement depending on the type of the served data. The goal of each SBS $i$ is to allocate its transmission power on each channel to serve its requests of a total size $l_{i}$ during a limited fraction of time that is constrained by the required time for the WUs to transmit their content successfully.

We define $p_{i}^{\min }=\left[p_{i 1}^{\min }, \ldots, p_{i C}^{\min }\right]$ as the minimum required transmit power of an SBS $i$ over all the channels to meet the delay constraint, given the power alloca- 
tion of other SBSs over the same unlicensed channels. The goal of each SBS is to minimize its transmit power over the unlicensed channels while accounting for the served WUs over the same channels. More formally, this problem can be defined as follows:

$$
\begin{aligned}
& p_{i}^{\min }=\arg \min _{p_{i} \in \mathbb{R}_{+}^{C}} \sum_{c=1}^{C} p_{i c}\left(p_{-i}\right), \\
& \text { subject to } \frac{l_{i c}}{w_{c} \log \left(1+\gamma\left(p_{i c}, p_{-i c}\right)\right)} \leq T_{\max }-t_{w c}, \\
& \sum_{c} p_{i c} \leq p_{i}^{\max }, \\
& p_{i c} \leq p_{c}^{\max }, \quad c \in \mathcal{C},
\end{aligned}
$$

where $p_{-i}$ is the power allocation of all other SBSs except SBS $i$ over all the channels $c \in \mathcal{C}$, and $l_{i c}$ is the amount of traffic that SBS $i$ wants to transmit over the unlicensed channel $c$. The power allocation $p_{i c}$ of SBS $i$ over each channel $c$ can be obtained by solving the following problem:

$$
\begin{aligned}
p_{i c}= & \underset{\left\{p_{i c}\right\}}{\arg \min _{c=1}} \sum_{i c}^{C} p_{i c}\left(p_{-i}\right), \\
\text { s.t. } \quad & R_{i c}=w_{c} \log \left(1+\gamma\left(p_{i c}, p_{-i c}\right)\right) \geq \frac{l_{i c}}{T_{\max }-t_{w c}}, \\
& \sum_{c} p_{i c} \leq p_{i}^{\max }, \\
& p_{i c} \leq p_{c}^{\max },
\end{aligned}
$$

where $p_{c}^{\max }$ is the maximum transmit power that is allowed over the unlicensed channel $c$, and $t_{w c}$ is the fraction of time the WUs require to transmit their packets successfully over the selected unlicensed channel $c . R_{i c}$ is the mean achievable rate by the LTE users served by SBS $i$ over the unlicensed channel $c$ as defined in (3) and can be rewritten as:

$$
R_{i c}=\omega_{c} \log \left(1+\frac{p_{i c}\left|h_{i c}\right|^{2}}{N+\sum_{l \in \mathcal{S} \backslash i} p_{l c}\left|h_{l c}\right|^{2}}\right),
$$

where the noise plus interference term from the WUs $N=\sigma^{2}+\sum_{j \in \mathcal{W}} \alpha_{j c} p_{j c}\left|h_{j c}\right|^{2}$, can be seen as a constant, which is independent of the power allocation of the SBSs. This is because the WUs define choose their channels in an a the leaders' game and the SBSs respond to these actions by determining their power allocation. We consider the noise plus interference term from WUs as a given term $N$ in (6) because the interference from the WUs transmitting over the same channel $c$ as the SBS $i$ is a constant, in the considered power allocation problem.

The power allocation problem (5a) is equivalent to the following problem: 


$$
\begin{aligned}
p_{i c}= & \underset{\left\{p_{i c}\right\}}{\arg \min _{c}} \sum_{i c} \\
\text { s.t. } & w_{c} \log \left(1+\gamma\left(p_{i c}, p_{-i c}\right)\right)=\frac{l_{i c}}{T_{\max }-t_{w c}}, \\
& \sum_{c} p_{i c} \leq p_{i}^{\max } \\
& p_{i c} \leq p_{c}^{\max }
\end{aligned}
$$

where equality holds for the rate requirements in the first constraint in (7).

This problem can be formulated as a noncooperative game given by:

$$
\mathcal{G}\left(t_{w}\right)=\left\{\mathcal{S},\left\{\mathcal{P}_{i}\right\}_{i \in \mathcal{S}},\left\{v_{i}\left(p_{i}, p_{-i}\right)\right\}_{i \in \mathcal{S}}\right\},
$$

where the set of SBSs $\mathcal{S}$ corresponds to the set of players, $v_{i}\left(p_{i}, p_{-i}\right)$ is the cost function per SBS and given by:

$$
v_{i}\left(p_{i}, p_{-i}\right)=\sum_{c \in \mathcal{C}} p_{i c}
$$

and $\mathcal{P}_{i}$ is the strategy set of SBS $i$ given by:

$$
\mathcal{P}_{i}\left(p_{-i}\right)=\left\{p_{i c} \in \mathbb{R}_{+}: \sum_{c \in \mathcal{C}} p_{i c} \leq p_{i}^{\max }, p_{i c} \leq p_{c}^{\max }, \frac{l_{i}}{w \log \left(1+\gamma\left(p_{i}, p_{-i}\right)\right)} \leq T_{\max }-t_{w}, \forall c \in \mathcal{C}\right\},
$$

and $v_{i}\left(p_{i}, p_{-i}\right)$ is the cost function of SBS $i$ that corresponds to the transmitted power as defined by the objective function (7). Due to the dependence between the player's $i$ strategy set $\mathcal{P}_{i}\left(p_{-i}\right)$ on other players' strategies $p_{-i}$, the solution of the game $\mathcal{G}$ is the Debreu equilibrium also known as the generalized Nash equilibrium [13], which can be defined as follows:

Definition 1. A strategy profile $p^{D E}$ is a Debreu equilibrium (DE) of the game $\mathcal{G}\left(t_{w}\right)$ if, for all the SBSs $i \in \mathcal{S}$, we have $p_{i}^{D E} \in \mathcal{P}_{i}\left(p_{-i}\right)$ with

$$
v_{i}\left(p_{i}^{D E}, p_{-i}^{D E}\right) \leq v_{i}\left(p_{i}, p_{-i}^{D E}\right)
$$

for all $p_{i} \in \mathcal{P}\left(p_{-i}^{D E}\right)$.

When all the SBSs except SBS $i$ select their transmit power strategies $p_{-i}$, the best choice of SBS $i$ consists in a selected strategy from the best-response strategies set $\mathcal{B}\left(p_{-i}\right)$ which is given by:

$$
\mathcal{B}_{i}\left(p_{-i}\right)=\underset{p_{i} \in \mathcal{P}_{i}\left(p_{-i}\right)}{\arg \min } v_{i}\left(p_{i}, p_{-i}\right) .
$$

Thus, the DEs can be derived by solving the fixed point problem (12) for all the $S$ SBSs. The resulting set of DEs might be empty as it might contain many DEs. Therefore, to analyze the hierarchical game, we first need to establish the existence 
and uniqueness of a $\mathrm{DE}$ in the formulated generalized noncooperative game. The time constraint bound in this case:

$$
w_{c} \log \left(1+\gamma\left(p_{i c}, p_{-i c}\right)\right)=\frac{l_{i c}}{T_{\max }-t_{w c}} .
$$

Thus, the minimum required transmission power of an SBS $i$ to serve its requests without exceeding the time limit is given by:

$$
p_{i c}^{*}=\min \left\{p_{i c}^{\max }, p_{i c}^{D E}\right\}
$$

where,

$$
p_{i c}^{D E}=\underset{p_{i c} \in \mathbb{R}^{+}}{\arg \min } v_{i}\left(p_{i c}, p_{-i}\right) .
$$

The following proposition provides the closed-form solution of the optimal power allocation and shows that this DE is unique.

Proposition 1. The DE power allocation for SBS i over channel c to its served LTE users is given by:

$$
p_{i c}^{D E}=\frac{N}{\left|h_{i c}\right|^{2}} \cdot \frac{1-2^{-\alpha_{i c}}}{\sum_{j=1}^{S} 2^{-\alpha_{j c}}-S+1} .
$$

Given the single SBS power constraint $p_{i}^{\max }$ and the power constraint on each unlicensed channel $c$, the rate requirement is only feasible if the following conditions are met.

Proposition 2. The DE power allocation $p_{i c}$ is feasible under the power constraints if and only if,

$$
\sum_{c} \frac{N}{\left|h_{i c}\right|^{2}} \cdot \frac{1-2^{-\alpha_{i c}}}{\sum_{j=1}^{S} 2^{-\alpha_{j c}}-S+1} \leq p_{i}^{\max },
$$

and,

$$
\max _{1 \leq j \leq S}\left(\frac{N\left(1-2^{-\alpha_{j c}}\right)}{p_{c}^{\max }\left|h_{i c}\right|^{2}}\right)+S-1<\sum_{j=1}^{S} 2^{-\alpha_{j c}}<S .
$$

Given the existence and the uniqueness of the DE for the power allocation game on the unlicensed channels, the goal of the WUs is to choose the unlicensed channels over which they serve their traffic. Next, we formulate and solve this problem as the high-level, leaders game.

\subsection{Leaders' Game}

Given the power allocation at the SBSs on each channel, the WUs have to choose the channels over which they transmit their traffic. This WUs-channels allocation problem can be formulated as a house allocation problem with existing tenants [11]. The house allocation problem with existing tenants is one-sided matching that is composed of a set of houses and a number of agents that want to rent a house while some of the houses are already occupied and their tenants may choose to participate 
or not in the assignment process. Similarly, some agents may already have a house while others do not. In our context, the WUs are the agents and the unlicensed channels correspond to the houses. The unlicensed allocation game can be defined as a tuple $\mathcal{P}=\left(\mathcal{S}, \mathcal{C} \cup\left\{c_{0}\right\},\left\{\kappa_{w c}\right\}_{\forall w \in \mathcal{W}, c \in \mathcal{C}},\left\{\prec_{w}\right\}_{w \in \mathcal{W}}, \mu\right)$ with each element defined as follows:

- The set $\mathcal{W}$ of WUs represents the set of players.

- The set $\mathcal{A}=\mathcal{C} \cup\left\{c_{0}\right\}$ of unlicensed channels is the set of actions that can be selected by each of the WUs, where $c_{0}$ corresponds to the case in which none of the unlicensed channels in chosen by the considered WU.

- $v_{w c}$ is the utility of WU $w$ when serving its traffic over unlicensed channel $c$.

- $\prec_{w}$ is the preference relation of the WUs. The preference relation $\prec_{w}$ is transitive and complete. We use $c \prec_{w} c^{\prime}$ to denote that WU $w$ prefers to serve its content over channel $c$ than serving it over channel $c^{\prime}$ for $c \neq c^{\prime}$.

- $\mu$ is the result of the actions selected by all the WUs.

The house allocation problem is a one-sided matching in which only the WUs have preferences over the channels while the unlicensed channels do not participate in the game by taking strategic decisions. This characteristic captures the fact that unlicensed spectrum is free and can be accessed by any user and thus, there does not exist any entity that can act on behalf of the unlicensed bands. The outcome of the house allocation problem with existing tenants can be defined as follows.

Definition 2. A matching between the WUs and unlicensed bands problem $\mu$ is a mapping from the set $\mathcal{W} \cup \mathcal{C}$ into the set $\mathcal{W} \cup \mathcal{C}$ such that for every $w \in \mathcal{W}$ and $c \in \mathcal{C}:$ i) $\mu^{-1}(w)$ is contained in $\mathcal{C}$ and $\mu(c)$ is contained in $\mathcal{W}$, ii) $\left|\mu^{-1}(w)\right| \leq 1$ for all $w \in \mathcal{W}$, iii) $|\mu(c)| \leq q_{c}$ for all $c \in \mathcal{C}$, iv) $c \in \mu^{-1}(w)$ if and only if $w \in \mu(c)$, where $q_{c}$ is the maximum number of WUs that can be served over channel $c$.

The value of $q_{c}$ is not predefined at the channels and depends on the amount of LTE traffic that each WU decides to serve over that channel as well as the amount of traffic that each WU decides to serve over the unlicensed channels. Definition 2 states that a WU $w$ can only select one unlicensed band $\mu^{-1}(w)$ while an unlicensed band $c$ can serve multiple WUs $\mu(c)$, depending on its capacity and the WiFi traffic load. Before setting the assignment of the WUs to the unlicensed channels, each WU needs to specify its preferences over the unlicensed channels based on its utility function. The externalities in the formulated matching problem appear in the throughput of a given WU that depends on the assigned users to each channel.

The goal of each $\mathrm{WU} w$ is to serve its content within the time duration $T_{\max }$. The utility of a $\mathrm{WU} w$ when transmitting over channel $c$ is given by:

$$
v_{w c}\left(c, p_{c}, \mu(c)\right)=T_{\max }-\hat{t}_{i c}\left(p_{c}\right)-t_{w c}\left(\mu(c), S_{c}\right),
$$

where $p_{c}=\left[p_{1 c}, \ldots, p_{S c}\right]$ is the transmit power of all the SBSs over channel $c$, and $t_{w c}\left(\mu(c), S_{c}\right)$ is given in (1). Assuming all the SBSs transmit at the same time, $\hat{t}_{i c}$ is the maximal duration during which the SBSs transmit and is given by: 


$$
\hat{t}_{i c}\left(p_{c}\right)=\underset{t_{i c}^{\prime}}{\arg \max }\left\{t_{i c}^{\prime}=\frac{l_{i}}{w_{c} \log \left(1+\gamma_{c}\left(p_{c}\right)\right)}, \forall i \in \mathcal{S}_{c}\right\},
$$

where $\mathcal{S}_{c}$ is the set of SBSs that decide to transmit over the unlicensed channel $c$ and $t_{i c}^{\prime}$ is the fraction of time during which an SBS $i$ uses channel $c$.

From (19), we can see that the utility of a WU $w$ not only depends on the set of WUs $\mu(c)$ that are assigned to channel $c$, but also on the interference generated by other SBSs transmitting over the same channel. Based on the defined utility function, each WU can define its preference relation $\prec_{w}$ over the set of channels $\mathcal{C}$, such that for any two channels $c, c^{\prime} \in \mathcal{C}$ with $c \neq c^{\prime}$, and two matchings $\mu, \mu^{\prime} \in \mathcal{W} \times \mathcal{C}$, $s \in \mu(c), w \in \mu^{\prime}\left(c^{\prime}\right)$ :

$$
(\mu, c) \prec_{w}\left(\mu^{\prime}, c^{\prime}\right) \Leftrightarrow v_{w c}\left(a_{-w}, \mu, S_{c}\right)<v_{w c^{\prime}}\left(a_{-w}, \mu^{\prime}, S_{c}\right),
$$

To solve the matching problem, we are interested in finding a desirable matching outcome that is Pareto optimal and in the core that can be defined as follows.

Definition 3. A matching $\mu$ is Pareto optimal if there does not exist another matching $\mu^{\prime}$ under which, at least one of the WUs can improve it utility while none of the WUs will degrade their utility under matching $\mu^{\prime}$ compared to matching $\mu$.

Definition 4. A matching $\mu$ is in the core of the one-sided matching $(\mathcal{W}, \mathcal{C}, \prec$

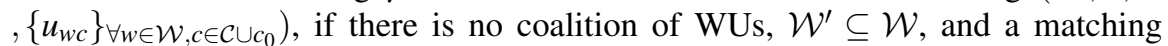
$\mu^{\prime}$ such that: i) $\mu^{\prime-1}(W) \in\left\{c_{0}\right\}_{\forall i \in \mathcal{W}^{\prime}}$ for all $s \in \mathcal{W}^{\prime}$, ii) $\mu^{-1}(w) \preceq_{w} \mu^{\prime-1}(w)$ for all $w \in \mathcal{W}^{\prime}$, iii) $\mu^{-1}(w) \prec_{w} \mu^{\prime-1}(w)$ for some $w \in \mathcal{W}^{\prime}$.

In the formulated problem, the channels can serve multiple WUs while in the original house allocation problem only one agent can be assigned to a given house. Thus, the existing algorithms do not account for the externalities that appear in the utility function (19) of the WUs as it depends on the WUs that are assigned to each of the channels. Moreover, the existing top trading cycle algorithm [11] for solving such problems is centralized and cannot be applied for the assignment of WUs to the unlicensed channels in which the algorithms should be decentralized due to the large number of access points. Next, we propose a new decentralized algorithm to solve the formulated one-sided matching game with externalities.

\subsubsection{Proposed Algorithm}

In the proposed algorithm, we solve the conflict between the WUs that want to access the same channel, by ranking the WUs randomly. Following the defined order, if a WU is not associated to its most preferred unlicensed channel that allows it to strictly increase its current utility, the WU $w_{0}$ sends a request to one of the WUs that is assigned to its most preferred channel called $w_{1}$. Upon receiving a request, if WU $w_{1}$ is not matched to its most preferred unlicensed channel, it sends a request to a $\mathrm{WU} w_{2}$ that is assigned to its most preferred channel. It also includes a list that contains the $\mathrm{WU} w_{1}$ from which it received a request and $\mathrm{WU} w_{0}$ that has sent a request to $\mathrm{WU} w_{1}$, thus, including all the WUs that lead to the initial requesting WU. At the end of the requests process, each WU checks the existence of a cycle 
in the received list from its requesting WUs. Once all the WUs emit their requests, the WUs that have initiated the requests procedure, check if a cycle is detected in the list they receive. If so, the WU with the highest order accepts the requests of its preceding WU in the list and transmit the list by removing itself from it. Each WU in the cycle does the same thing until the last WU of the list. Then, all the WUs that belong to a cycle leave the matching game and the WUs update their preferences list based on the remaining WUs. The assignment process is then repeated among the remaining WUs and their associated channels.

Theorem 1. The WUs-channels assignment that results from the proposed algorithm is in the core.

Theorem 2. The outcome of the proposed matching algorithm is the unique matching in the core.

Theorem 3. The proposed algorithm is Pareto efficient.

\section{Conclusions}

In this paper, we have addressed the problem of coexistence over unlicensed bands. In particular, we have formulated a multi-game in which the WUs are considered as leaders that have priority when accessing the unlicensed bands, and the SBSs as followers. In the leaders game, the WUs select the unlicensed channel using which they transmit their content while anticipating the SBSs' possible reactions. In the followers game, the SBSs follow the leaders decisions and respond to it by determining their transmit power over each of the channels.

\section{References}

1. F. Liu, E. Bala, E. Erkip, M. C. Beluri, and R. Yang, "Small cell traffic balancing over licensed and unlicensed bands," IEEE Transactions on Vehicular Technology, vol. PP, no. 99, 2015.

2. R. Zhang, M. Wang, L. X. Cai, Z. Zheng, X. S. Shen, and L.-L. Xie, "LTE-unlicensed: The future of spectrum aggregation for cellular networks," IEEE Wireless Communications, vol. 22, no. 3, pp. 150-159, 2015.

3. H. Zhang, X. Chu, W. Guo, and S. Wang, "Coexistence of wi-fi and heterogeneous small cell networks sharing unlicensed spectrum,” IEEE Communications Magazine, vol. 53, no. 3, pp. 158-164, 2015.

4. Y. Li, F. Baccelli, J. G. Andrews, T. D. Novlan, and J. C. Zhang, "Modeling and analyzing the coexistence of wi-fi and lte in unlicensed spectrum," arXiv preprint arXiv:1510.01392, 2015.

5. K. Hamidouche, W. Saad, and M. Debbah, "A multi-game framework for harmonized lte-u and wifi coexistence over unlicensed bands," IEEE Wireless Communications Magazine, Special Issue on LTE in Unlicensed Spectrum, vol. 32, no. 6, pp. 1065-1082, 2014.

6. M. Chen, W. Saad, and C. Yin, "Echo state networks for self-organizing resource allocation in LTE-U with uplinkdownlink decoupling," arXiv preprint arXiv:1601.06895, 2016.

7. Y. Gu, Y. Zhang, L. X. Cai, M. Pan, L. Song, and Z. Han, "Exploiting student-project allocation matching for spectrum sharing in LTE-unlicensed," in IEEE Global Communications Conference, 2015, pp. 1-6.

8. X. Kang, Y.-K. Chia, S. Sun, and H. F. Chong, "Mobile data offloading through a third-party wifi access point: An operator's perspective," IEEE Transactions on Wireless Communications, vol. 13, no. 10, pp. 5340-5351, 2014.

9. Q. Chen, G. Yu, H. M. Elmaghraby, Z. Ding et al., "Embedding lte-u within wi-fi bands for spectrum efficiency improvement," arXiv preprint arXiv:1607.04729, 2016.

10. O. Galinina, A. Pyattaev, S. Andreev, M. Dohler, and Y. Koucheryavy, "5G multi-RAT LTE-WiFi ultra-dense small cells: performance dynamics, architecture, and trends," IEEE Journal on Selected Areas in Communications, vol. 33 , no. 6, pp. 1224-1240, 2015.

11. A. Abdulkadiroğlu and T. Sönmez, "House allocation with existing tenants," Journal of Economic Theory, vol. 88, no. 2, pp. 233-260, 1999.

12. W. Lingzhi, H. Cunqing, Z. Rong, and N. Rui, "Online channel selection and user association in high-density wifi networks," in International Conference on Communications (ICC), 2015, pp. 1571-1576.

13. G. Debreu, "A social equilibrium existence theorem," Proceedings of the National Academy of Sciences of the United States of America, vol. 38, no. 10, p. 886, 1952. 